\title{
Antes NIC 39 ahora NIIF 9: nuevos desafíos para los contadores
}

\section{Daniela del Pilar Rodríguez Díaz}

\author{
Departamento Académico de Ciencias de la Gestión \\ Pontificia Universidad Católica del Perú
}

En este trabajo, se busca sintetizar las principales diferencias en la aplicación de la NIIF 9 «Instrumentos Financieros» a implementarse de forma obligatoria en el Perú desde el inicio del ejercicio 2018 con respecto a la actual NIC 39 «Instrumentos Financieros: Reconocimiento y Medición», específicamente en clasificación y medición de las partidas de activos financieros. Además, se realiza un análisis práctico aplicado a instrumentos financieros del rubro de la banca de inversión.

Palabras clave: Banco de inversión, activos financieros, pasivos financieros, NIIF 9, NIC 39, modelo de negocio, clasificación, medición

\section{Before IAS 39, Now IFRS 9: New Challenges for Financial Accountants}

In this paper we will resume the main differences between the application of IFRS 9 «Financial Instruments» to be imperatively implemented in Peru since the commencement of the exercise 2018 with regards to the current IAS 39 «Financial Instruments: Recognition and Measurement», focusing in the classification and measurement of financial assets. Furthermore, a practical analysis will be applied to financial instruments in the investment banking industry.

Keywords: Investment bank, financial assets, financial liabilities, IFRS 9, IAS 39, business model, classification, measurement

\section{Antes da IAS 39, agora IFRS 9: Novos desafios para contadores financeiros}

Neste artigo, retomaremos as principais diferenças entre a aplicação da Normas Internacionais de Relatório Financeiro (IFRS) 9 «Instrumentos Financeiros» a implementar de forma imperativa no Peru desde o início do exercício de 2018 no que se refere ao atual Normas internacionais de contabilidade (IAS) 39 «Instrumentos Financeiros: Reconhecimento e Medição», focado em A classificação e mensuração de ativos financeiros. Além disso, uma análise prática será aplicada aos instrumentos financeiros no setor de banca de investimento.

Palavras-chave: Banco de investimento, ativos financeiros, passivos financeiros, IFRS 9, IAS 39, modelo de negócios, classificação, medição 


\section{Introducción}

Recuerdo los años 2005-2007, cuando cursaba mi época universitaria en la Facultad de Administración y Contabilidad en la PUCP, y revisaba detenidamente mis libros y apuntes sobre las Normas Internacionales de Contabilidad (NIC). Particularmente, me sentí atraída por aquellas normas vinculadas a instrumentos financieros. La más emblemática para mí es la mítica $-\mathrm{y}$, a veces, complicada - Norma Internacional de Contabilidad 39 «Instrumentos Financieros: Reconocimiento y Medición» ${ }^{1}$. Si aún no terminamos de entenderla después de años de aplicación, una nueva norma viene en camino para consolidar y cambiar algunos de los supuestos que venimos aplicando en los estados financieros de las empresas.

Luego de varios borradores, la versión final de la Norma Internacional de Información Financiera 9 o NIIF 9 (International Financial Reporting Standard 9 o IFRS 9, por sus siglas en inglés, en adelante «la Norma») fue promulgada por el Consejo de Normas Internacionales de Contabilidad (International Accounting Standards Board o IASB por sus siglas en inglés) en julio del año 2014 con el objetivo de dirigir el registro de los instrumentos financieros y, sobre todo, la forma en que se miden después de su reconocimiento inicial. Abarca tres temas importantes: la clasificación y medición de instrumentos financieros, el deterioro de activos financieros y la contabilidad de coberturas. Es de aplicación obligatoria para períodos que empiecen en o después del 1 de enero de $2018^{2}$, por lo cual las empresas deben encontrarse en pleno proceso de implementación. Dicha implementación impacta no solamente en el área contable, sino en las áreas involucradas en el modelo de negocio de cada compañía. Al respecto, EY indica que la versión final de la Norma «recopila todas las fases del proyecto de instrumentos financieros y sustituye a la NIC 39: Instrumentos Financieros: Reconocimiento y Medición y a todas las versiones previas de la NIIF 9» (2015, p. 9). Localmente, en el Perú, se aprueba la versión final de la NIIF 9 mediante la resolución 056-2014-EF/30 3 del Consejo Normativo de Contabilidad, publicada el 12 de noviembre de 2014.

Debido a que el tema es amplio, este artículo se enfoca en el primer tema mencionado previamente: la clasificación y medición de instrumentos financieros. Estos incluyen los activos y pasivos financieros. Si bien abordaremos estos últimos, se dará mayor énfasis al tratamiento de los activos financieros debido a la materialidad de los cambios en los mismos.

Cabe acotar que, en cuanto a activos financieros, la NIC 39 prioriza la intención y capacidad de las entidades, más que el análisis contractual de los instrumentos y que el modelo de negocio para los cuales son obtenidos; esta es la novedad de la NIIF 9. Se debe tener en cuenta los hechos y las circunstancias particulares de cada una de las inversiones, y cumplir con los requisitos de la NIIF 9 para cada una de las categorías de inversiones. Antes de empezar, se indican algunas definiciones importantes.

\footnotetext{
Incluye a la NIC 32 «Instrumentos Financieros: Presentación» y la NIIF 7 «Instrumentos Financieros: Revelaciones».

2 Cabe anotar que se puede aplicar anticipadamente.

3 Esta resolución oficializa las modificaciones a la NIC 16 «Propiedades, Planta y Equipo», y a la NIC 41 «Agricultura», la versión final de la NIIF 9 «Instrumentos Financieros» y la NIIF 15 «Ingresos de Actividades Ordinarias Procedentes de Contratos con Clientes».
} 


\section{Definiciones}

- Con reciclaje, se refiere a que los resultados generados por la venta o liquidación de los instrumentos se reclasificarán en los resultados del período, y, como consecuencia, la ganancia o pérdida generada se reclasificará desde resultados no realizados (cuenta patrimonial) a los resultados del ejercicio.

- FVTPL corresponde a los instrumentos medidos a valor razonable con cambios en resultados (del inglés, Fair Value through Profit or Loss).

- FVOCI corresponde a los instrumentos medidos a valor razonable con efecto en otro resultado integral (del inglés Fair Value through Other Comprehensive Income)

- OCI refiere a otro resultado integral (Other Comprehensive Income), que constituye una cuenta del patrimonio.

- La prueba de SPPI consiste en la prueba de flujos de caja contractuales que representan únicamente pagos de principal e intereses (del inglés Solely Payments of Principal and Interest).

- Sin reciclaje, se refiere a que los resultados generados por la venta o liquidación de los instrumentos nunca van a colocarse en el estado de resultados, y, como consecuencia, la ganancia o pérdida generada se reclasificará directamente de los resultados no realizados a los resultados acumulados de la compañía (reclasificación entre cuentas patrimoniales).

- SPPI alude a los flujos de caja contractuales que representan únicamente pagos de principal e intereses (de inglés Solely Payments of Principal and Interest).

\section{Activos financieros}

La NIIF 9 enfoca la clasificación de los activos financieros sobre la base de los flujos de caja de cada instrumento y en los modelos de negocio bajo los que se administran dichos activos de acuerdo con la cascada de decisión siguiente:

Desde la línea punteada del gráfico hacia abajo, se detallan los aspectos a tomar en cuenta para realizar una adecuada medición posterior de instrumentos de deuda y de patrimonio, los cuales se detallan a continuación.

\subsection{Instrumentos de deuda}

La medición bajo el costo amortizado es aplicable a los activos financieros representativos de deuda que satisfacen dos condiciones según la NIIF 9 (IASB, 2014):

- Las características de los flujos de efectivo contractuales tienen que representar el reembolso del principal y de los intereses sobre el principal, marco en el que el interés es definido como la compensación por el valor del dinero en el tiempo y el riesgo de crédito (en esencia, esta condición requiere que los términos del instrumento sean simples o del inglés plain vanilla).

- Los activos financieros son gestionados en un modelo de negocio cuyo objetivo es recaudar los flujos de efectivo contractuales (en oposición al objetivo de realizar el valor razonable mediante la venta).

Asimismo, la NIIF 9 indica que la medición a valor razonable con cambios en otro resultado integral (FVOCI) es aplicable para los activos financieros representativos de deuda que satisfagan dos condiciones (IASB, 2014): 
Figura 1. Resumen de clasificación de instrumentos financieros de deuda y patrimonio

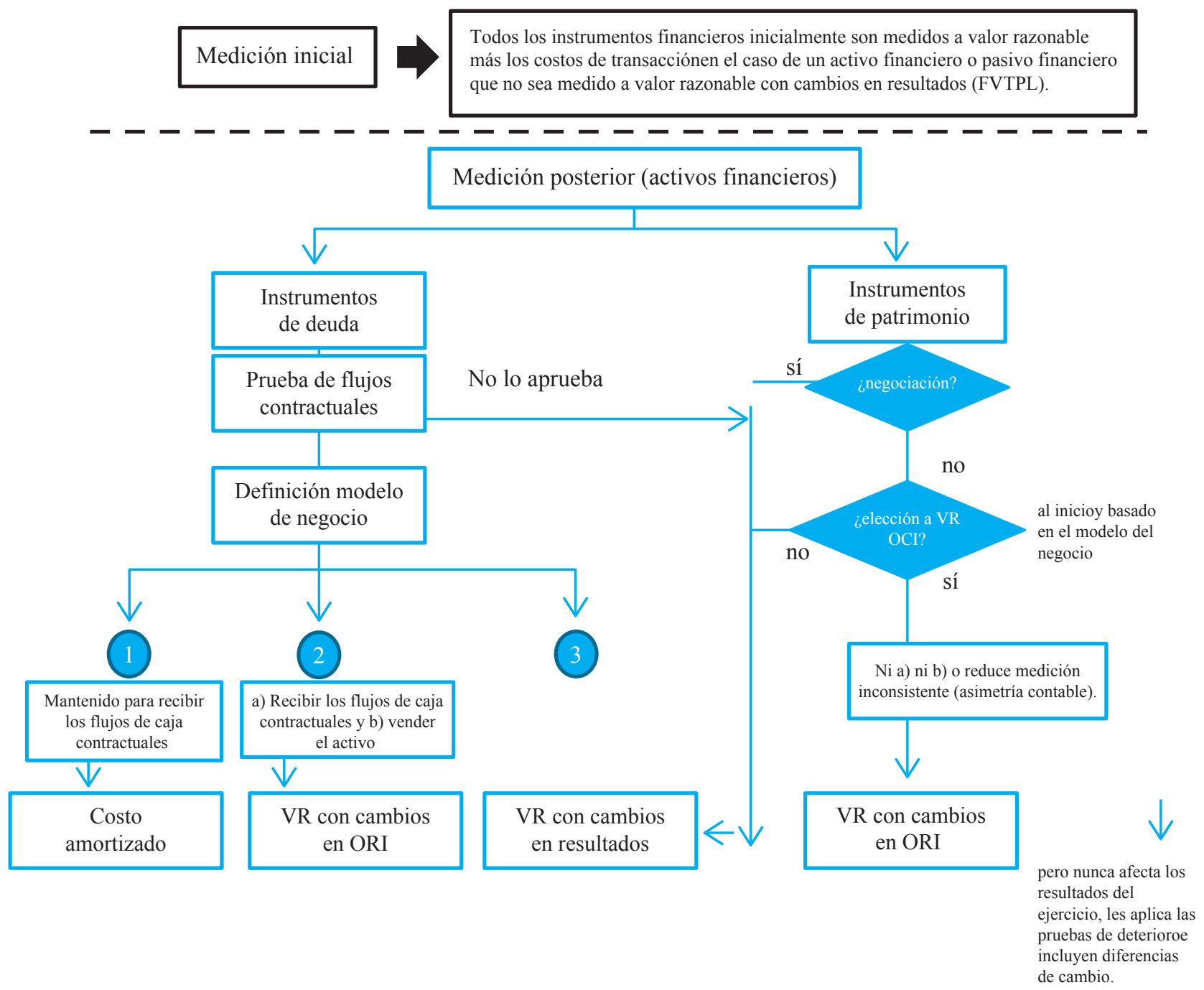

Fuente: IASB (2014). Elaboración propia.

- Las características de los flujos de efectivo contractuales tienen que representar el reembolso de principal e intereses sobre el principal (la misma condición de los flujos de efectivo contractuales que para los activos a costo amortizado mencionados anteriormente).
- Los activos deben encontrarse en un modelo de negocio cuyo objetivo sea tanto recaudar los flujos de efectivo contractuales como vender los activos financieros (esto último es particularmente relevante para las empresas que cuentan con portafolios de liquidez). 
Los activos financieros representativos de deuda que no satisfagan las condiciones indicadas anteriormente para la medición a costo amortizado o a valor razonable con cambios en otro resultado integral (FVOCI) tienen que ser medidos a valor razonable con cambios en resultados (FVTPL)

Ahora, si se cumplen ambas pruebas, la empresa también tiene la capacidad de designar un activo financiero como a valor razonable con cambios en resultados si al hacerlo se reduce o elimina una medición o reconocimiento que sea inconsistente: una asimetría contable.

\subsubsection{Ejemplo de asimetría contable}

La asimetría contable se produce cuando existen activos y pasivos que se encuentran directamente relacionados, pero tienen bases de medición distintas si los analizamos de forma individual. Por ejemplo, podría darse el caso de que el activo cumple con todas las características para ser medido a valor razonable con cambios en otro resultado integral (FVOCI), mientras que el pasivo solo puede ser medido a valor razonable con cambios en resultados (FVTPL). Sin embargo, debido a que estos instrumentos están directamente relacionados, tener dos clasificaciones distintas genera que —en lugar de que los resultados generados por la medición a valor razonable del activo y el pasivo se compensen- el patrimonio presente una volatilidad por el activo, mientras el estado de resultados tenga una volatilidad separada por el pasivo. Este es un ejemplo de una asimetría contable y, para evitarla, la Norma brinda la opción de designar un activo que puede ser medido a valor razonable con cambios en otro resultado integral (FVOCI) como a valor razonable con cambios en resultados (FVTPL). De esta manera, es posible evitar este tipo de asimetrías o descalces.

\subsection{Instrumentos de patrimonio}

Los instrumentos de patrimonio son aquellos instrumentos clasificados como a valor razonable con cambios en resultados (FVTPL), a menos que se elija de forma irrevocable clasificarlos en la categoría de instrumentos a valor razonable con cambios en otro resultado integral (FVOCI). Esta elección se realiza sobre la base de cada instrumento de patrimonio.

En la clasificación a valor razonable con cambios en otro resultado integral (FVOCI), todos los cambios de valor razonable — con exclusión de los dividendosque constituyan un retorno de la inversión serán reportados en otro resultado integral, específicamente dentro de la cuenta patrimonial «Resultados No Realizados», y no se transferirán a resultados del período en ningún caso — por ejemplo- con motivo de su venta, por lo que tampoco están sujetos a pruebas de deterioro. No obstante, la entidad puede transferir la ganancia o pérdida no realizada hacia resultados acumulados cuando se ejecute la venta del instrumento, pero esta reclasificación no afectaría el patrimonio.

\subsection{1. ¿A qué se refiere la norma cuando indica que el término «con» $y$ «sin» reciclaje?}

Este término hace referencia a los instrumentos clasificados como a valor razonable con cambios en otro resultado integral (FVOCI) y sus lineamientos para reclasificar los resultados reconocidos en el patrimonio vía la cuenta resultados no realizados, pues son diferentes para instrumentos de deuda y de patrimonio, de acuerdo con el siguiente detalle: 
Figura 2. Resumen de impactos posteriores en instrumentos medidos al FVOCI

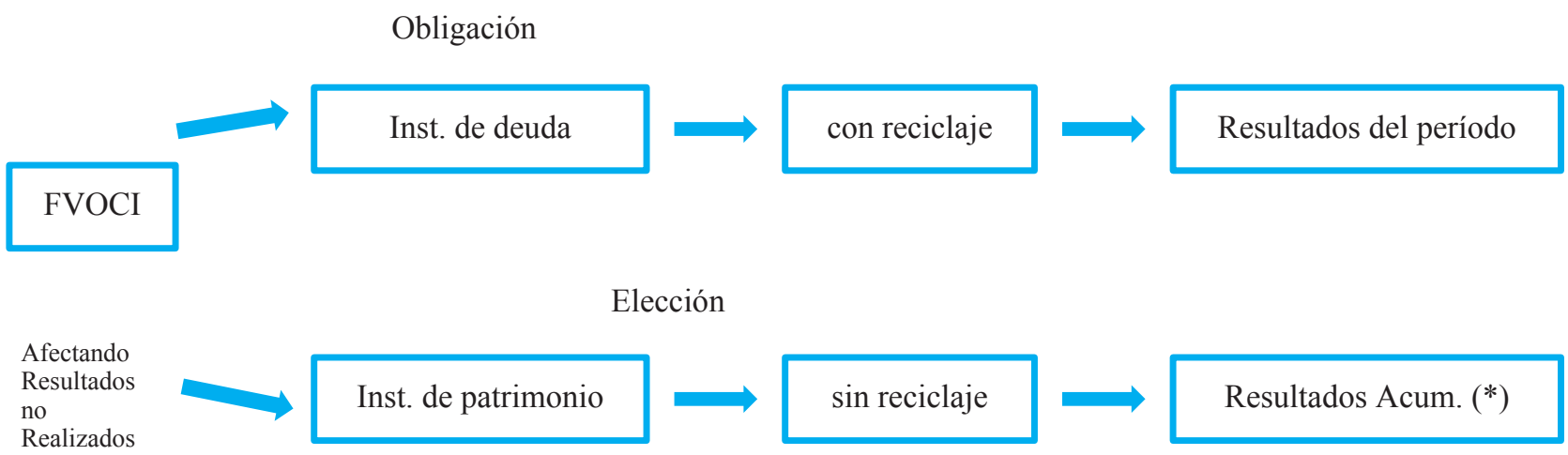

(*) Solo se reconoce el resultado los ingresos por dividendos de instrumentos de patrimonio.

\section{Los desafíos en la implementación de la categoría de inversiones}

Respecto a los nuevos criterios establecidos por la NIIF 9 para clasificar las inversiones de una entidad, el portafolio de inversiones que actualmente se encuentra en la categoría de inversiones disponibles para la venta, según la NIC 39 (IASB, 2012c), es la que tendrá mayor complejidad en el análisis, debido a lo siguiente:

- La categoría de disponible para la venta en la actual NIC 39 es una clasificación residual y una elección sin restricciones; en contraposición, bajo la NIIF 9, la clasificación de mantener o vender, que tiene un tratamiento similar, debe ser el reflejo de las características contractuales del instrumento y de un modelo de negocio demostrado con hechos y circunstancias; por lo tanto, no es una categoría residual ni una elección.

- Solo los instrumentos de deuda típicos, como los bonos, se clasificarán a FVOCI (tratamiento similar a la actual clasificación de disponible para la venta), debido a que tendrán que pasar la prueba de las características de flujo de efectivo contractuales, y el modelo de negocio debe ser aquel que genera ganancias mediante la recepción de flujos de caja contractuales y la venta de esos activos.

En los dos puntos anteriores, se introducen dos conceptos nuevos respecto de la NIC 39. La prueba de modelo de negocio y prueba de características de flujo de efectivo contractuales (prueba de SPPI).

\section{Prueba de modelo de negocio}

Los modelos de negocio establecidos por la NIIF 9 son tres (IASB, 2014):

(a) Mantenidos con la finalidad de obtener flujos de caja contractuales

(b) Mantenidos con la finalidad de obtener flujos de caja contractuales y realizar ventas los activos involucrados

(c) Modelo de negocio cuyo fin no es ni (a) ni (b) 
Las compañías deben diferenciar los portafolios o estrategias que usan en la gestión de sus inversiones, y asociar cada uno de esos portafolios a un determinado modelo de negocio según los tres indicados (a, b y c). Este es determinado por el personal clave de la Gerencia respecto de la manera como se gestionan los activos financieros y cómo su desempeño es reportado hacia ellos. Esta prueba se realiza a nivel de grupos de activos financieros y refleja cómo se administran con la finalidad de generar flujos de caja. Esta evaluación se realiza sobre la base de los escenarios probables que se espera ocurran de manera razonable. Por ejemplo, un portafolio con inversiones a vencimiento se consideraría como dentro del modelo (a), debido a que el objetivo será mantener esos instrumentos hasta su vencimiento y, por lo tanto, los rendimientos obtenidos serán originados por los flujos de caja contractuales del instrumento (a través de intereses devengados), mientras que un portafolio de negociación (de trading por sus siglas en inglés) correspondería al modelo (b), puesto que el objetivo principal consiste en obtener ganancias por las ventas de los instrumentos a corto plazo. Este análisis y diferenciación son necesarios, debido a que el modelo de negocio va a definir el tipo de medición adecuada para cada portafolio. Es importante mencionar que una o más estrategias pueden estar asociadas a un mismo modelo de negocio. El modelo de negocio para administrar activos financieros es una cuestión de hecho y es normalmente observable a través de actividades particulares que una compañía realiza para lograr sus objetivos. Se necesita utilizar el juicio para evaluar el modelo de negocio para la administración de activos financieros, y dicha evaluación no es determinada por un único factor o actividad. Se deben considerar todas las evidencias que se encuentran disponibles en la fecha de evaluación. Algunos ejemplos que nos indica la NIIF 9 son los siguientes:
- Cómo el desempeño del modelo de negocio y de los activos financieros mantenidos dentro del mismo son evaluados y reportados al personal clave de la Gerencia

- Los riesgos que afectan el desempeño de los modelos de negocio, y los activos financieros mantenidos dentro del mismo y la manera en la que se administran estos riesgos

- Cómo los gerentes de los negocios son remunerados

- La frecuencia y el valor de venta esperado de los instrumentos financieros, principalmente, para el modelo de «Obtener flujos de caja contractuales y realizar ventas de los activos involucrados»

Vale la pena aclarar que la evaluación del modelo de negocio sobre el cual se realiza una inversión aplica tanto para instrumentos de deuda como de patrimonio.

\section{Prueba de los flujos de caja contractuales (prueba de SPPI)}

Un tema importante incorporado en la NIIF 9 es la prueba de flujos de caja contractuales, que representan únicamente pagos de principal e intereses (del inglés Solely Payments of Principal and Interest o SPPI), pues esta requiere un análisis exhaustivo sobre los flujos de caja contractuales por cada instrumento. La definición de interés es la contraprestación por el valor temporal del dinero y por el riesgo de crédito (incluidos otros riesgos, costos de préstamos y un margen de ganancia) asociado con el importe principal presente durante un período determinado de tiempo (IASB, 2014).

La prueba es efectiva solo cuando la variabilidad de los flujos de caja contractuales surge como resultado de mantener el retorno del inversionista bajo un acuerdo básico de préstamo, en el cual la aplicación de la tasa de interés efectiva proporciona información útil. 
El acuerdo básico de préstamo consiste en obtener el activo financiero original, de acuerdo con lo siguiente: el inversionista busca obtener un rendimiento que compensa principalmente el valor del dinero en el tiempo y el riesgo de crédito. Adicionalmente, podría incluir el riesgo de liquidez, costos relacionados con la posesión del activo financiero por un período de tiempo (costos administrativos, por ejemplo) y un margen de utilidad, criterios que deben ser revisados a profundidad.

\section{Un caso particular: ejemplo de un banco de inversión}

En el rubro de la banca de inversión, se mantienen activos financieros a valor razonable con cambios en resultados y disponibles para la venta, de acuerdo con la NIC 39. El portafolio de inversiones se gestiona considerando las siguientes estrategias:

- Estrategias de negociación (trading): Tiene el objetivo de adquirir inversiones y venderlas en el corto plazo con la finalidad de generar márgenes de rentabilidad en función de la apreciación de las inversiones.

- Estrategias propias del negocio: Estas se encuentran vinculadas con su rol de intermediario financiero en la compra y venta de inversiones, que consiste en realizar operaciones con activos financieros que se registran por cuenta propia. Dichas operaciones incluyen dos tipos:

- Operaciones formadoras de mercado (market making): En estas operaciones, se adquiere por cuenta propia inversiones (principalmente, acciones) de un emisor en función de un contrato pactado con este y, posteriormente, estas son vendidas en el corto plazo con el objetivo de darle liquidez a dicho instrumento financiero.
El objetivo de estas operaciones en la Bolsa de Valores de Lima (BVL), por ejemplo, es generar una menor brecha (spread) entre los precios de compra y venta de instrumentos financieros (de patrimonio y/o de deuda), e impulsar así el número de operaciones (Chiarella, 2016).

- Operaciones de facilitación (facilitation): Suponen la recepción informal de una solicitud de un cliente para comprar un instrumento o un grupo de ellos en un futuro cercano (días), para lo cual se adquieren posiciones de acuerdo al requerimiento del cliente, pero en cuenta propia, con la finalidad de aprovechar las condiciones más ventajosas del mercado. Luego, dichos instrumentos serán vendidos al cliente mediante la formalización de una solicitud de compra de valores, principalmente, con clientes institucionales. Este concepto incluye las ganancias por operaciones de arbitraje de instrumentos financieros.

- Operaciones para mantener «inversiones regulatorias»: Estas son participaciones en acciones adquiridas con el objetivo de obtener acceso a mecanismos centralizados para el desarrollo de operaciones propias del negocio de intermediación financiera y son de índole regulatoria, así como participaciones en entidades que se consideran clave.

Debido a la naturaleza del negocio de intermediación financiera, no se mantienen inversiones a mediano o largo plazo. Vinculadas estas estrategias con el modelo de negocio, en el caso de instrumentos de deuda, las inversiones no se mantienen para obtener flujos de efectivo contractuales ni para venderlas, por lo que se aplicaría el criterio «a valor razonable con cambios en resultados» o FVTPL. En el caso de instrumentos de patrimonio, se llevarían «a valor razonable con cambios en resultados» o se podría ejercer la opción irrevocable 
de medirlas «a valor razonable con cambios en otro resultado integral» (o FVOCI). Esta opción es similar a la forma como se encuentran actualmente registrados estos instrumentos bajo el alcance de la NIC 39, dado que se tratan como inversiones disponibles para la venta, lo cual afecta el patrimonio de la compañía.

Para colocar un ejemplo, tenemos los siguientes datos de un instrumento representativo de deuda que un banco de inversión mantiene en su cartera propia correspondiente a la estrategia de negociación o trading indicada anteriormente.
Durante el año 2016, se han realizado las siguientes cinco compras de este instrumento, y se calcula la ganancia por el período de tenencia o devengo del capital y la fluctuación del valor de mercado, dado que es un instrumento de deuda que se compró a cargo de la mesa de negociación (trading) bajo el modelo de negocio que suponía vender el activo, pero no necesariamente para obtener los flujos de caja del mismo, pues el objetivo no es tenerlo hasta el vencimiento de acuerdo a la estrategia de inversión. Por lo tanto, encaja en la tercera definición de la Figura 1; es decir, deberá medirse a valor razonable con cambios en resultados.

Tabla 1. Ejemplo de datos de un instrumento de deuda definido para la estrategia de negociación de un banco de inversión

\begin{tabular}{|l|l|}
\hline \multicolumn{1}{|c|}{ Datos } & \multicolumn{1}{c|}{ Descripción } \\
\hline Instrumento de deuda & Certificado del Banco Central de Reserva del Perú (BCRP) \\
\hline Tipo & Al descuento ${ }^{4}$ \\
\hline Moneda & PEN \\
\hline Plazo & 340 días \\
\hline Fecha de emisión & $23 / 09 / 2015$ \\
\hline Fecha de vencimiento & $09 / 03 / 2017$ \\
\hline Fecha de valorización & $31 / 12 / 2016$ \\
\hline Precio de mercado & $99,2064($ al 31.12.2016) \\
\hline
\end{tabular}

Tabla 2. Detalle de compras realizadas y valorización del instrumento al 31 de diciembre 2016 (en soles - S/)

\begin{tabular}{|c|c|c|c|c|c|c|}
\hline Nro. & Fecha operación & Cantidad & Precio compra & Costo (a) & Plazo remanente & TEA (\%) \\
\hline 1 & $17 / 03 / 2016$ & 5.000 .000 & 95,2319 & 4.761 .595 & 289 & $5,12 \%$ \\
\hline 2 & $31 / 03 / 2016$ & 5.000 .000 & 95,4838 & 4.774 .190 & 275 & $5,04 \%$ \\
\hline 3 & $18 / 10 / 2016$ & 600.000 & 98,3237 & 589.942 & 74 & $4,44 \%$ \\
\hline 4 & $18 / 10 / 2016$ & 400.000 & 98,3237 & 393.295 & 74 & $4,44 \%$ \\
\hline 5 & $15 / 11 / 2016$ & 9.500 .000 & 98,6774 & 9.374 .353 & 46 & $4,36 \%$ \\
\hline
\end{tabular}

\footnotetext{
4 Se refiere a que se paga al vencimiento el valor nominal; es decir, no hay cupones. Por ello, los intereses devengados implícitos equivalen a la diferencia entre el costo inicial y el costo amortizado antes del cobro del certificado.
} 


\begin{tabular}{|c|c|c|c|c|}
\hline \multirow{2}{*}{ Nro. } & \multicolumn{4}{|c|}{ Al 31.12.2016 Precio de mercado: 99,2064\% } \\
\cline { 2 - 5 } & Costo amortizado (b) & Valor razonable (c) & Intereses implícitos (b-a) & Fluctuación (c-b) \\
\hline 1 & 4.953 .687 & 4.960 .319 & 192.092 & 6632 \\
\hline 2 & 4.954 .400 & 4.960 .319 & 180.210 & 5919 \\
\hline 3 & 595.162 & 595.238 & 5220 & 76 \\
\hline 4 & 396.775 & 396.826 & 3480 & 51 \\
\hline 5 & 9.424 .851 & 9.424 .606 & 50.498 & -246 \\
\hline & 20.324 .876 & 20.337 .307 & 431.501 & 12.431 \\
\hline
\end{tabular}

\begin{tabular}{|l|c|c|c|}
\hline \multicolumn{2}{|c|}{ Al 31 de diciembre 2016 (en S/) } & Efecto & Cuenta contable (estado de resultados) \\
\hline Costo inicial (a) & 19.893 .375 & & \\
\hline Costo amortizado (b) & 20.324 .876 & 431.501 & Ingresos financieros (intereses implícitos) \\
\hline Valor razonable (c) & 20.337 .307 & 12.431 & Ingresos por fluctuación de valor razonable \\
\hline
\end{tabular}

\section{Aspectos importantes a considerar cuando se tomen las opciones de clasificación y medición}

\subsection{Aspectos importantes en cuanto a la clasificación inicial de instrumentos de patrimonio}

\section{Sobre la elección de uso de la medición «A valor razonable con cambios en otro resultado integral (FVOCI)»}

En la medición inicial, la empresa debe definir los instrumentos de patrimonio desde el punto de vista del emisor, por los cuales optará de manera irrevocable a clasificarlos como «a valor razonable con cambios en otro resultado integral (FVOCI)». Esta debe ser realizada por cada instrumento y genera que, con excepción de los dividendos que se reconocen en resultados, las ganancias y pérdidas asociadas -incluidas las diferencias de cambio- sean reconocidas en otro resultado integral dentro de las cuentas patrimoniales (exactamente en la cuenta resultados no realizados). Las mismas conforman otra cuenta patrimonial $y$, entonces, el patrimonio permanece exactamente igual. Estas inversiones no son partidas monetarias, $y$, por tanto, las ganancias o pérdidas que se registren en el otro resultado integral (patrimonio) incluyen los resultados por diferencias de cambio de la moneda extranjera correspondiente. En el ejemplo, este tratamiento contable aplicaría para las inversiones en instrumentos de patrimonio que representan una inversión regulatoria, de acuerdo con la definición descrita anteriormente, la cual incluye inversiones mantenidas para propósitos no financieros, por ejemplo, cuando existe un requerimiento que implica mantener una determinada inversión si una entidad ofrece sus servicios en un mercado en particular, como la Cámara de Compensación Electrónica S.A. o la Bolsa de Valores de Lima (BVL). Estas acciones (instrumentos de patrimonio) adquiridas para fines regulatorios cumplen con el modelo de negocio de mantener la inversión, pero no se vuelven estratégicas en el tiempo, sino que se adquirieron como tales desde un inicio y deben estar clasificadas como a valor razonable con cambios en otro resultado integral (FVOCI). 
De acuerdo con lo establecido en la NIC 32, los instrumentos de patrimonio incluyen básicamente acciones comunes y algunos tipos de acciones preferenciales (no cualquier inversión en renta variable), los cuales deben ser evaluados desde el punto de vista del emisor del instrumento de acuerdo con la siguiente definición:

Según la NIC 32, un instrumento de patrimonio se considera como tal, solo si se cumplen las siguientes dos (2) condiciones: 1) El instrumento no incluye la obligación contractual para el emisor de: (i) entregar efectivo u otro activo financiero a otra entidad, ni (ii) intercambiar activos o pasivos financieros con otra entidad bajo condiciones que son potencialmente desfavorables para el emisor. 2) Si el instrumento será o puede ser liquidado por el emisor a través de sus propios instrumentos de patrimonio, entonces es: (i) un instrumento no derivado que incluye obligaciones no contractuales para el emisor de entregar un número variable de sus propios instrumentos de patrimonio o, (ii) un derivado que será liquidado solo a través del intercambio del emisor de una cifra determinada de efectivo u otro activo financiero por un número determinado de sus propios instrumentos de patrimonio (IASB, 2012b)

Esto implica que un instrumento de patrimonio es cualquier contrato que ponga de manifiesto una participación residual en los activos de una entidad después de restar todos sus pasivos.

Algunos ejemplos de instrumentos que son presentados desde el punto de vista del emisor como patrimonio y generan una rentabilidad variable en un banco de inversión son los siguientes:
- Las participaciones en fondos mutuos ${ }^{5}$ o fondos negociables en el mercado ${ }^{6}$ que inclusive pueden invertir únicamente en instrumentos de deuda son considerados fondos abiertos, pues aceptan nuevos inversionistas después de su constitución y los inversionistas previamente existentes tienen la opción de dejar el fondo en cualquier momento (existen nuevas suscripciones y rescates). El valor al que nuevos ingresantes invierten en el fondo o al que los que se retiran dejan el fondo generalmente se basa en el valor razonable de los activos. Los inversionistas, entonces, ingresan y se retiran del fondo a partir de su valor razonable y es poco probable que los flujos de caja de una inversión en dicho fondo correspondan únicamente a pagos de principal e intereses. Los inversionistas pueden ingresar o salir de los fondos basándose en el valor razonable de los activos subyacentes, más allá de solo recibir flujos contractuales de caja.

- Un segundo caso es el de las cuotas de participación en fondos cerrados o fondos de capital privado (private equity), fondos de cobertura (hedge funds) y otros similares. Las participaciones en fondos cerrados o fondos de capital privado, casos en los que el tenedor tiene el derecho de devolver las participaciones al fondo a cambio de su participación proporcional en los activos netos (es decir, cuando cuenta con una opción de venta), cumplen con los requisitos para ser clasificadas como instrumentos de patrimonio desde la perspectiva del fondo, pero, desde la perspectiva del tenedor, estas participaciones no cumplen con la definición de instrumentos

5 Los fondos mutuos son patrimonios autónomos que se conforman con los aportes de dinero de una gran cantidad de personas. Están administrados por las sociedades administradoras de fondos mutuos y supervisados por la Superintendencia del Mercado de Valores.

6 También, son conocidos como fondos cotizados en Bolsa o Exchange Traded Funds (ETF, por sus siglas en inglés). Son fondos de inversión cuyas participaciones se negocian en bolsa, se liquidan y negocian de la misma manera que las acciones (Benito, 2011). 
de patrimonio de la NIC 32. Sin embargo, el IASB ha comentado que esto puede darse en la realidad y es aceptada su clasificación como instrumentos de patrimonio.

- Un tercer caso es el de las cuotas de participación en fondos de pensiones.

\subsection{Aspectos importantes en cuanto a la clasificación inicial de los instrumentos de deuda}

\section{Elección de uso de la medición «A valor razonable con cambios en resultados» (FVTPL)}

Se pueden medir de esta manera si al hacerlo se elimina o reduce significativamente una inconsistencia en la medición o reconocimiento (asimetría contable) que pudiera surgir. Por ejemplo, en las empresas aseguradoras, esta designación aplicaría para las inversiones financieras que respaldan pasivos por seguros provenientes de productos. Los resultados de estas inversiones (ganancias o pérdidas) se asignan directamente a los tenedores de los contratos de seguros, que son los que asumen el riesgo de inversión. Como consecuencia de ello, los pasivos relacionados a estos contratos son de igual importe que los activos que los respaldan, neto de las comisiones que cobre la empresa aseguradora por la administración de estos contratos. Los activos financieros que son administrados sobre la base de valor razonable, entonces, siempre serán clasificados como con cambios en resultados (FVTPL) y no se necesita ejercer una opción por estos instrumentos.

Finalmente, para clasificar un instrumento de deuda, se debe determinar el modelo de negocio de un portafolio o grupo de activos financieros, el cual debe reflejar la manera como la entidad administra dichos activos con el fin de generar flujos de efectivo. La frecuencia de las ventas es un aspecto importante para la determinación de un modelo de negocio. El punto más importante es que se requiere considerar la expectativa de las ventas futuras y considerar a las ventas pasadas solo como una fuente de evidencia. Por otro lado, la Norma requiere que, si un número de ventas infrecuente se ejecuta en un portafolio y el valor de estas ventas son más que significativas de forma individual o en conjunto, la entidad deberá evaluar si tales ventas son consistentes con el objetivo de un modelo de negocio establecido solo para la cobranza de flujos de caja contractuales. Entonces, las empresas tienen una tarea: determinar qué características definen a una venta como «infrecuente» y/o "significativa», pues no se establecen los porcentajes específicos para estos criterios.

\section{Reclasificaciones y cambios en el modelo de negocio}

Bajo la nueva NIIF 9, las reclasificaciones entre categorías de instrumentos de patrimonio no están permitidas y, en el caso de instrumentos de deuda, solo están permitidas por cambios en el modelo de negocio, aunque se espera que sean esporádicos, dado que se debe fundamentar un cambio en la dirección de la entidad como resultados de cambios internos o externos a ella, los cuales deben ser materiales, no estacionarios y demostrables ante terceros. Utilizando el ejemplo del banco de inversión, se ha explicado que este utiliza un modelo de negociación o de trading, el cual no podría fácilmente convertirse en un banco de ahorro que utiliza un modelo de negocio de «crear y mantener inversiones». Si las operaciones cambian en este sentido, el banco de inversión tendría que, como parte de su evaluación del cambio de modelo de negocio, analizar la reclasificación de su portafolio desde valor razonable con cambios en resultados 
(con una estrategia de negociación) a costo amortizado (estrategia de mantener o buy and hold) o, en su defecto, a valor razonable con cambios en otro resultado integral (FVOCI).

Sin embargo, según la NIIF 9, no se consideran cambios en el modelo de negocio los siguientes casos:

- Cambios en la intención relacionada con un activo financiero: Por ejemplo, una disminución en la frecuencia de las ventas de activos mantenidos por el banco de inversión para negociar no implica que haya cambiado su modelo de negocio y, por lo tanto, no conlleva a una reclasificación de categoría. Sin embargo, el cambio en la intención de inversión en determinados activos que son significativos en valor y a nivel transaccional puede suponer que se evalúe si su modelo de negocio cambió.

- Desaparición temporal de un mercado particular de un activo financiero: Por ejemplo, si el banco de inversión tuviera instrumentos con períodos de iliquidez o que dejarán de cotizar en mercados activos, esto no implica un cambio en la estrategia del modelo de negocio de trading. La iliquidez de un activo no conlleva a que una entidad modifique sus estrategias de gestión de portafolios de inversiones. Puntualmente, se puede hablar de las transacciones formadoras de mercado o de market making, a través de las cuales se busca brindar liquidez a un instrumento de un determinado emisor que contrata este servicio. Los instrumentos se adquieren también bajo la premisa de negociarlos en el corto plazo y, aunque exista un período de liquidez en los mismos, el objetivo de adquirirlos no es mantenerlos, pues la característica del servicio está asociada a siempre estar disponible para comprar o vender el instrumento $y$, pesar de que no sea tan líquido, el modelo no cambia.

- Transferencia de activos financieros entre partes de la empresa con diferentes modelos de negocio: Si bien no aplica para el ejemplo del banco de inversión, debido a que el modelo de negocio vigente corresponde únicamente a negociación o trading, en el caso que existieran más modelos de negocio en la compañía, las transferencias de activos entre dichos modelos de negocio no representarían un cambio de modelo cuando estas se dan por condiciones específicas y no por cambios en la estrategia de toda la cartera en que se haya designado el activo. ¿Por qué esto no aplica al ejemplo del banco de inversión? Se debe considerar que existe un tratamiento específico para las inversiones regulatorias designadas así desde un inicio. Estas son medidas a valor razonable con cambios en otro resultado integral (FVOCI) y no se vuelven regulatorias o estratégicas en el tiempo, sino que son designadas de esta manera desde el inicio, y la estrategia actual de inversiones corresponde solo al modelo de negociación. Tomando esto en cuenta, sería muy improbable que existan transferencias entre la cartera de inversiones para fines regulatorios, y la cartera de inversiones para negociación y sus subcategorías.

Por último, la reclasificación se debe reconocer de forma prospectiva y los hechos deben haber ocurrido antes a la reclasificación A continuación, se adjunta una tabla con los impactos de las reclasificaciones de los activos financieros.

Llegado a este punto, resulta útil realizar un comparativo de los principales cambios en la NIIF 9 respecto de la NIC 39. 
Tabla 3. Impactos de las reclasificaciones sobre la base de la NIIF 9

\begin{tabular}{|c|c|c|}
\hline Desde & Hacia & Impacto \\
\hline Costo amortizado & $\begin{array}{l}\text { Valor razonable con } \\
\text { cambios en resultados } \\
\text { (FVTPL) }\end{array}$ & $\begin{array}{l}\text { Cualquier efecto positivo o negativo por las diferencias se reconocerá en } \\
\text { el resultado del periodo. El valor razonable se mide en la fecha de reclasi- } \\
\text { ficación. }\end{array}$ \\
\hline $\begin{array}{l}\text { Valor razonable con cambios } \\
\text { en resultados (FVTPL) }\end{array}$ & Costo amortizado & $\begin{array}{l}\text { El valor razonable en la fecha de reclasificación se transforma en su nuevo } \\
\text { valor en libros bruto; por lo tanto, no existe impacto en los resultados. }\end{array}$ \\
\hline Costo amortizado & $\begin{array}{l}\text { Valor razonable con } \\
\text { cambios en otro resultado } \\
\text { integral (FVOCI) }\end{array}$ & $\begin{array}{l}\text { Cualquier efecto positivo o negativo por las diferencias se reconocerá en } \\
\text { otro resultado integral. El valor razonable se mide en la fecha de reclasifi- } \\
\text { cación }\end{array}$ \\
\hline $\begin{array}{l}\text { Valor razonable con cambios } \\
\text { en otro resultado integral } \\
\text { (FVOCI) }\end{array}$ & Costo amortizado & $\begin{array}{l}\text { Los resultados acumulados anteriormente reflejados en el patrimonio se } \\
\text { eliminarán y se ajustarán contra el valor razonable del activo financiero en } \\
\text { la fecha de reclasificación dentro de los resultados del ejercicio. }\end{array}$ \\
\hline $\begin{array}{l}\text { Valor razonable con cambios } \\
\text { en otro resultado integral } \\
\text { (FVOCI) }\end{array}$ & $\begin{array}{l}\text { Valor razonable con } \\
\text { cambios en resultados } \\
\text { (FVTPL) }\end{array}$ & $\begin{array}{l}\text { Los resultados acumulados anteriormente reflejados en el patrimonio } \\
\text { (resultados no realizados) se reclasificarán hacia el resultado del periodo } \\
\text { como un ajuste. }\end{array}$ \\
\hline
\end{tabular}

Fuente: IASB (2014). Elaboración propia.

\section{Tabla 4. Comparativo NIC 39 y NIIF 9}

\begin{tabular}{|c|c|c|c|}
\hline Tema & Criterio & NIC 39 & NIIF 9 \\
\hline $\begin{array}{l}\text { Parámetro para } \\
\text { la clasificación } \\
\text { de instrumentos }\end{array}$ & & $\begin{array}{l}\text { Refiere a la intención y a la capacidad de } \\
\text { la entidad de mantener el instrumento } \\
\text { financiero, ya sea hasta su vencimiento o } \\
\text { para su negociación a corto plazo. }\end{array}$ & $\begin{array}{l}\text { La clasificación se encuentra basada en un modelo de negocio } \\
\text { y las características de los flujos de caja contractuales de cada } \\
\text { instrumento financiero. }\end{array}$ \\
\hline $\begin{array}{l}\text { Inversiones } \\
\text { disponibles para } \\
\text { la venta }\end{array}$ & & $\begin{array}{l}\text { Es una categoría residual, pues no son ni } \\
\text { mantenidos para su negociación ni a ven- } \\
\text { cimiento. No tiene restricciones. }\end{array}$ & $\begin{array}{l}\text { Ya no existe. Sin embargo, su medición (a valor razonable } \\
\text { con cambios en otro resultado integral) se aplica cuando se } \\
\text { cumple uno de los modelos de negocio para instrumentos de } \\
\text { deuda y de patrimonio. }\end{array}$ \\
\hline $\begin{array}{l}\text { Instrumentos } \\
\text { de deuda }\end{array}$ & $\begin{array}{l}\text { Forma de } \\
\text { medición } \\
\text { posterior }\end{array}$ & $\begin{array}{l}\text { Costo amortizado para instrumentos } \\
\text { mantenidos hasta el vencimiento } \\
\text { A valor razonable con cambios en resul- } \\
\text { tados } \\
\text { A valor razonable con cambios en otros } \\
\text { resultados integrales para inversiones dis- } \\
\text { ponibles para la venta }\end{array}$ & $\begin{array}{l}\text { Costo amortizado si el objeto del negocio es cobrar los flujos } \\
\text { de caja contractuales que son el principal e intereses sobre el } \\
\text { instrumento } \\
\text { A valor razonable con cambios en otro resultado integral si } \\
\text { el objetivo del modelo de negocio consiste en la obtención } \\
\text { de los flujos de caja contractuales del instrumento (deben } \\
\text { ser únicamente por el pago del principal e intereses sobre el } \\
\text { mismo) y también para vender los activos } \\
\text { A valor razonable con cambios en resultados si el modelo de } \\
\text { negocio no cumple con ninguna de las características antes } \\
\text { mencionadas y si los flujos de caja contractuales no pasan la } \\
\text { prueba de SPPI (ver Figura 1) } \\
\text { A valor razonable con cambios en resultados con el funda- } \\
\text { mento de que, con ello, se elimina una asimetría contable (ver } \\
\text { "Ejemplo de asimetría contable») }\end{array}$ \\
\hline
\end{tabular}




\begin{tabular}{|c|c|c|c|}
\hline Tema & Criterio & NIC 39 & NIIF 9 \\
\hline & $\begin{array}{l}\text { Reclasifi- } \\
\text { caciones }\end{array}$ & $\begin{array}{l}\text { Están permitidas en circunstancias espe- } \\
\text { cíficas, y las ganancias o pérdidas son } \\
\text { tratadas de manera diferente dependiendo } \\
\text { del movimiento entre las categorías. }\end{array}$ & $\begin{array}{l}\text { Están permitidas a partir del cambio en el modelo de negocio, } \\
\text { a nivel del total de los instrumentos o portafolios afectados, los } \\
\text { cuales deben estar sustentados, aprobados por la Gerencia y } \\
\text { deben ser poco frecuentes (pues el modelo de negocio es estable). }\end{array}$ \\
\hline \multirow[t]{4}{*}{$\begin{array}{l}\text { Instrumento de } \\
\text { patrimonio }\end{array}$} & \multirow{3}{*}{$\begin{array}{l}\text { Forma de } \\
\text { medición } \\
\text { posterior }\end{array}$} & $\begin{array}{l}\text { A valor razonable con cambios en resulta- } \\
\text { dos si son mantenidos para negociación. }\end{array}$ & A valor razonable con cambios en resultados \\
\hline & & $\begin{array}{l}\text { A valor razonable con cambios en otro } \\
\text { resultado integral para la clasificación de } \\
\text { inversiones disponibles para la venta. Las } \\
\text { ganancias y/o pérdidas registradas en los } \\
\text { resultados no realizados (cuenta patri- } \\
\text { monial) se reclasifican a resultados del } \\
\text { período. }\end{array}$ & $\begin{array}{l}\text { Opción sin cambio de designar el instrumento en el momento } \\
\text { inicial como a valor razonable con cambios en otro resultado } \\
\text { integral con la condición de que el instrumento no sea man- } \\
\text { tenido para negociación. Esta opción es llamada como "sin } \\
\text { reciclaje», pues los resultados generados no se reconocerán en } \\
\text { los resultados del período en el caso de su enajenación y/o } \\
\text { baja, sino seguirán en otra cuenta patrimonial. Los dividen- } \\
\text { dos sí se registran en los resultados del ejercicio. }\end{array}$ \\
\hline & & $\begin{array}{l}\text { Si existen inversiones que no cotizan } \\
\text { en un mercado activo, se podrán medir } \\
\text { excepcionalmente al costo cuando el } \\
\text { valor razonable no pueda ser determi- } \\
\text { nado con confiabilidad. }\end{array}$ & $\begin{array}{l}\text { Se elimina la posibilidad de medir al costo inversiones de } \\
\text { patrimonio no cotizadas cuando el valor razonable no pueda } \\
\text { ser medido confiablemente. }\end{array}$ \\
\hline & $\begin{array}{l}\text { Reclasifi- } \\
\text { caciones }\end{array}$ & $\begin{array}{l}\text { Entre las categorías de valor razonable } \\
\text { con cambios en resultados y de dispo- } \\
\text { nible para la venta, las reclasificaciones } \\
\text { están permitidas. } \\
\text { Cuando se transfieren desde disponibles } \\
\text { para la venta, los resultados no realizados } \\
\text { se reconocen en los resultados del ejercicio. } \\
\text { Cuando se transfieren desde la categoría } \\
\text { de valor razonable con cambios en resul- } \\
\text { tados, no está permitido realizar ninguna } \\
\text { reversión de las ganancias o pérdidas rea- } \\
\text { lizadas a no realizadas. }\end{array}$ & $\begin{array}{l}\text { Entre las categorías de a valor razonable con cambios en resul- } \\
\text { tados hacia valor razonable con cambios en otros resultados } \\
\text { integrales, las reclasificaciones no están permitidas, debido } \\
\text { a que la elección de llevarlo a valor razonable con cambios } \\
\text { en otros resultados integrales se realiza en el reconocimiento } \\
\text { inicial del instrumento de manera irrevocable (ver Figura 1). }\end{array}$ \\
\hline
\end{tabular}

Fuente: IASB (2012c, 2014).

\section{Otras consideraciones}

Para cualquier empresa, es muy importante contar con cuentas contables específicas que permitan identificar los instrumentos de patrimonio adquiridos con fines regulatorios. En el caso de las participaciones en instrumentos de patrimonio (acciones) que no hayan sido adquiridas con un fin de negociación, tal como es el caso de las participaciones en la Bolsa de Valores de
Lima (BVL) o en la Bolsa de Comercio de Santiago (BCS), en el caso del banco de inversión, existe la opción irrevocable de registrar dichas inversiones como a valor razonable con cambios en resultados (FVTPL) o como a valor razonable con cambios en otro resultado integral (FVOCI). Esto es particularmente importante, pues uno de los requerimientos de la NIIF 9 consiste en revelar los activos y resultados generados por este tipo de inversiones cuando se ha optado por 
usar la clasificación FVOCI. Por ello, es importante mantener cuentas contables específicas en los estados financieros para este tipo de posiciones. Adicionalmente, es importante definir internamente dentro de los procesos de la compañía qué órgano interno será el encargado de aprobar la designación de instrumentos de patrimonio como a valor razonable con cambios en otro resultado integral (FVOCI) o qué área será la encargada de realizar la prueba de flujos de caja contractuales (SPPI). En el caso de bancos de inversión, se propone que sea el área de Riesgos conjuntamente con el área de Finanzas, y que los impactos de las clasificaciones iniciales y posteriores sean reportados al Comité de Activos y Pasivos (Alco) para su aprobación, que normalmente es la instancia especializada en temas financieros dentro de una entidad bancaria. En dicho comité, participa la Alta Dirección de la compañía, incluidos el gerente general y los directores, dado que es un tema de suma relevancia para los resultados e indicadores financieros de la entidad (como el ROE o ROA). Aún nos queda tiempo para revisar estas particularidades dentro de nuestras compañías; empecemos por conocer nuestro negocio.

\section{Referencias}

Benito, Antonio (2011). ETF'S (Exchanged traded funds) o fondos cotizados en Bolsa [videograbación]. Valencia: Universidad Politécnica de Valencia. Consulta: 10 de octubre 2016. https://www.youtube.com/watch?v=al Wx60TAUnI

Chiarella, Ramón (2016). Market makers en la BVL: Un menor spread impulsa el número de operaciones. Semana Económica. s/l, 3 de junio. Consulta: 10 de octubre 2016. http://semanaeconomica.com/article/mercadosy-finanzas/189804-market-makers-en-la-bvl-un-menor-spread-impulsa-el-numero-de-operaciones/
Consejo Normativo de Contabilidad (2014). Resolución 056-2014-EF/30. Lima, 6 de noviembre. Consulta: 10 de septiembre 2016. https://mef.gob.pe/index. php?option=com_content $\&$ view $=$ article $\&$ id $=1383 \& I$ temid $=101208 \&$ lang $=$ es

EY (2015). Situación de los distintos proyectos del IASB y del Comité de Interpretaciones de las NIIF. Actualización Contable, 10, 8-11. Consulta: 10 de setiembre 2016. http://www.ey.com/Publication/vwLUAssets/ Actualizacion-contable-marzo-2015/ \$FILE/EY-Actualizacion-Contable10.pdf

International Accounting Standard Board (IASB) (2012b). Norma Internacional de Contabilidad 32: Instrumentos Financieros: Presentación.

International Accounting Standard Board (IASB) (2012c). Norma Internacional de Contabilidad 39: Instrumentos Financieros: Reconocimiento y Medición de Estados Financieros.

International Accounting Standard Board (IASB) (2014). Norma Internacional de Información Financiera 9: Instrumentos Financieros.

Recibido: 25 de octubre de 2016 Aceptado: 03 de junio de 2017 daniela_rodri@hotmail.com 